\title{
Tiling the Plane with Permutations
}

\author{
Alexandre Blondin Massé ${ }^{1}$, Andrea Frosini², \\ Simone Rinaldi ${ }^{3}$, and Laurent Vuillon ${ }^{1}$ \\ ${ }^{1}$ Laboratoire de Mathématiques, Université de Savoie, CNRS UMR 5127, \\ 73376 Le Bourget du Lac \\ 2 Università di Firenze, Dipartimento di Sistemi e Informatica, \\ viale Morgagni 65, 50134 Firenze \\ 3 Università di Siena, Dipartimento di Matematica e Informatica, \\ Pian dei Mantellini 44, 53100 Siena
}

\begin{abstract}
A permutomino is a polyomino uniquely determined by a pair of permutations. Recently permutominoes, and in particular convex permutominoes have been studied by several authors concerning their analytical and bijective enumeration, tomographical reconstruction, and the algebraic characterization of the associated permutations 23. On the other side, Beauquier and Nivat [5] introduced and gave a characterization of the class of pseudo-square polyominoes, i.e. polyominoes that tile the plane by translation: a polyomino is called pseudo-square if its boundary word may be factorized as $X Y \bar{X} \bar{Y}$.

In this paper we consider the pseudo-square polyominoes which are also convex permutominoes. By using the Beauquier-Nivat characterization we provide some geometrical and combinatorial properties of such objects, and we show for any fixed $X$, each word $Y$ such that $X Y \bar{X} \bar{Y}$ is pseudo-square is prefix of an infinite word $Y_{\infty}$ with period $4|X|_{N}|X|_{E}$.

Some conjectures obtained through exhaustive search are also presented and discussed in the final section.
\end{abstract}

\section{Introduction}

Tiling the plane using polyominoes is a rather popular research topic, sometimes related with computational theory, mathematical logic and discrete geometry. This subject has been studied under different points of view: combinatorial properties of the tiles have been considered in order to count them or the patterns they produce in the plane, and, consequently, group them in classes presenting similar behaviors; a geometrical approach has been applied in order to find new characterizations of the tiles in terms of their shape or properties of their border; finally, also tomographical aspects of tilings have been studied with the aim of retrieving geometrical properties of a tiling from partial and sometimes inaccurate measurements of the local densities (see [6]).

Interesting results have been achieved by restricting the class of sets of tiles only to those having one single element. In particular Wijshoff and Van Leeuwen [8] considered the exact polyominoes (i.e. polyominoes which tile the plane by translation) and proved that the problem of recognizing them is decidable. In [5], 
Beauquier and Nivat studied the same problem from a purely geometrical point of view and they found a characterization of all the exact polyominoes by using properties of the words which describe their boundaries. In particular they stated that the boundary word coding these polyominoes shows a pattern $X Y Z \bar{X} \bar{Y} \bar{Z}$, called a pseudo-hexagon, where one of the variable may be empty in which case the pattern $X Y \bar{X} \bar{Y}$ is called a pseudo-square. Successively, in [1] the authors studied some combinatorial and enumeration problems on the pseudo-square convex polyominoes.

In this paper we relate pseudo-square polyominoes with the class of permutominoes. A permutomino of size $n$ is a polyomino determined by particular pairs $\left(\pi_{1}, \pi_{2}\right)$ of permutations of length $n$, such that $\pi_{1}(i) \neq \pi_{2}(i)$, for $1 \leq i \leq n$ (see, for instance, Fig. (4). An equivalent definition for a permutomino is that, for each abscissa (ordinate) between 1 and $n$, there is exactly one vertical (horizontal) side in the boundary with that coordinate.

Permutominoes can be viewed as special types of permutation diagrams, and they have been introduced by Kassel et al. [7] while studying some algebraic problems related with the $\widetilde{R}$-polynomials associated with a pair $\left(\pi_{1}, \pi_{2}\right)$ of permutations.

During the last years, a particular class of permutominoes, namely the class of convex permutominoes have been widely studied: in [3] the authors provide their enumeration according to the size, while in [2] the authors give a characterization of permutation defining convex permutominoes.

In this paper we consider the class of pseudo-square convex permutominoes (briefly, psc-permutominoes), i.e. polyominoes which are both pseudo-square and convex permutominoes. This problem raises interesting properties since it relates to combinatorics on words and classical problems on polyominoes. Moreover these objects are uniquely defined by permutations, thus they give us an effective way to tile the plane using permutations. Hence this problem shows strict relations with the vast combinatorics on pattern avoiding permutations [2].

In this paper we focus on the problem of establishing if, for a given word $X$, there is at least a word $Y$ which is compatible with $X$, that is, $X Y \bar{X} \bar{Y}$ represents a $p s c$-permutomino. Using the Beauquier-Nivat characterization, and the results from [1, we show that for any given $X$ there are at most two different words of minimal length $Y$ and $Y^{\prime}$ compatible with $X$. These words are such that $|Y|=\left|Y^{\prime}\right|$, and $Y^{\prime}$ can be obtained from $Y$ by means of renaming of words. Moreover, they individuate the growing direction of the permutomino, so we distinguish among the up and the down-growing direction of a $p s c$-permutomino and study these two cases separately.

In studying the $p s c$-permutominoes with up-growing direction, we prove that $X$ uniquely determines an infinite word $Y_{\infty}$, and that every word $Y$ which is up-compatible with $X$ (i.e. $X Y \bar{X} \bar{Y}$ is a psc-permutomino with up-growing direction) is a prefix of $Y_{\infty}$. Moreover we show that $Y_{\infty}$ has period $4|X|_{E}|X|_{N}$. The same results can be reformulated in the case of down-growing direction.

Several challenging problems remain open, and some conjectures obtained through exhaustive search are also presented and discussed in the final section. 


\section{Two Classes of Polyominoes}

In the plane $\mathbb{Z} \times \mathbb{Z}$ a cell is a unit square whose vertices have integer coordinates, and a polyomino is a finite connected union of cells having no cut point (see Fig. 1(a)). Polyominoes are defined up to translations, and we deal with polyominoes without "holes", i.e. polyominoes whose boundary is a single loop. A column (resp. row) of a polyomino is the intersection between the polyomino and an infinite strip of cells whose centers lie on a vertical (resp. horizontal) line. A polyomino is said to be column-convex (resp. row-convex) when its intersection with any vertical (resp. horizontal) line is connected. A polyomino is convex if it is both column and row convex (see Fig. 1(b,c)).

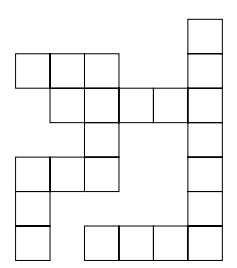

(a)

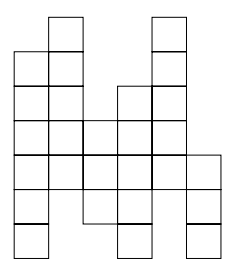

(b)

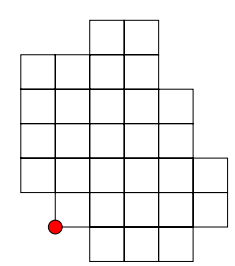

(c)

Fig. 1. A polyomino (a), a column-convex polyomino (b), a convex polyomino (c)

A particular subclass of the class of convex polyominoes consists of the parallelogram polyominoes, defined by two lattice paths that use north (vertical) and east (horizontal) unitary steps, and intersect only at their origin and extremity. These paths are commonly called the upper and the lower path (see Fig. 2).
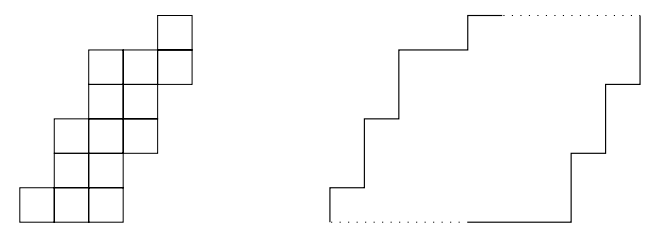

Fig. 2. A parallelogram polyomino, its upper and lower paths

The boundary of a polyomino can be conveniently represented by a boundary word defined on the alphabet $\Sigma=\{N, S, E, W\}$, where $N$ (resp. $E, S, W$ ) stands for the north (resp. east, south, west) unit step. The word representing a polyomino is obtained simply by following its boundary from a starting point in a clockwise orientation. For instance, the polyomino in Fig. 1(c), starting from the highlighted point, is represented by the word

$$
X=N W N N N N E E N E E S S E S S E S S W S W W W N W .
$$


Moreover, if $X=x_{1} x_{2} \ldots x_{r}$ is a word, where $x_{i} \in\{N, E, S, W\}$, then the complement $\bar{X}=\bar{x}_{r} \ldots \bar{x}_{2} \bar{x}_{1}$ is defined by $\bar{N}=S, \bar{S}=N, \bar{W}=E$, and $\bar{E}=W$. We define the length of a word $X$ by $|X|=r$, and by $|X|_{x_{i}}$ the number of occurrences of the letter $x_{i}$ in $X$. We observe that in any polyomino the length of the boundary word coincides with its perimeter.

\subsection{Polyominoes That Tile the Plane}

In [5], Beauquier and Nivat studied the class of exact polyominoes, i.e. polyominoes that tile the plane by translation. They found a characterization of all the exact polyominoes by using properties of the words which describe their boundaries. In particular they stated that the boundary words coding these polyominoes show a pattern $X Y Z \bar{X} \bar{Y} \bar{Z}$, called a pseudo hexagon, where one of the variable may be empty in which case the pattern $X Y \bar{X} \bar{Y}$ is called a pseudo-square.

In the rest of the paper, we will deal with pseudo-square convex polyominoes (briefly psc-polyominoes) As an example, the polyomino in Fig. 3 (a) is a pscpolyomino, and the decomposition of its boundary word is

$$
X=N N E N E E N, Y=E E \bar{N} E, \bar{X}=\bar{N} \bar{E} \bar{E} \bar{N} \bar{E} \bar{N} \bar{N} \text { and } \bar{Y}=\bar{E} N \bar{E} \bar{E} \text {. }
$$

We recall some properties about psc-polyominoes obtained in [1, which will be useful through the paper. We first consider pseudo-square parallelogram polyominoes. In this case we have:

Proposition 1. If $X Y \bar{X} \bar{Y}$ is a decomposition of the boundary word of a pseudosquare parallelogram polyomino, then $X Y$ encodes its upper path, and $Y X$ its lower path. Moreover, $X$ starts and ends with $N$, and $Y$ starts and ends with $E$.

For psc-polyominoes which are not parallelograms, we can have at most two decompositions which are strictly related to each other:

1. the first one is such that the path $X$ of the decomposition starts from the lowest point in the leftmost column and ends in the leftmost point in the uppermost row (see Figure $3(c)$ ), then it has the form $N^{+}(E \vee N)^{*} N^{+}$. The starting point of such decomposition is called $A$; we observe that also parallelogram polyominoes have this kind of decomposition;

2. the second one is such that the path $X$ starts from the uppermost point in the leftmost column and ends in the rightmost point in the uppermost row, (see Figures $3(b),(d)$ ). The starting point of such decomposition is called $B$.

We also observe that each psc-polyomino with a decomposition of the second type can be transformed - under an horizontal reflection - into another psc-polyomino whose decomposition is of the first type (note that the two polyominoes may coincide). So, without loss of generality we will only consider $p s c$-polyominoes of with a decomposition of the first type, starting from $A$. Hence, from now on, the word $X$ encoding the boundary of a generic psc-permutomino is assumed to have the form $N^{+}(E \vee N)^{*} N^{+}$. 


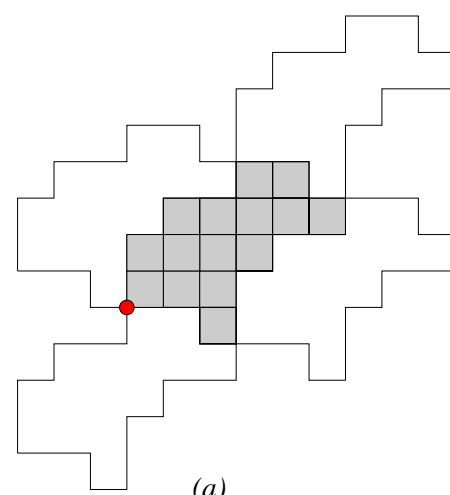

(a)

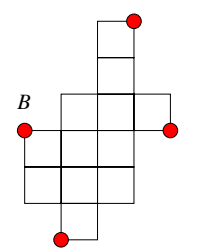

(b)

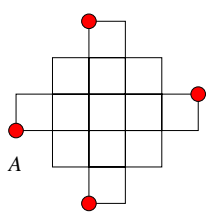

(c)

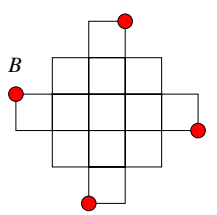

(d)

Fig. 3. (a) a psc-polyomino (with highlighted cells) having only a decomposition of the first type, and the corresponding tiling of the plane; (b) a psc-polyomino having one decomposition of the second type; in $(c),(d)$ a psc-polyomino admitting decompositions of the first and of the second type; the starting points are $A$ and $B$, respectively

\subsection{Polyominoes Determined by Permutations}

Let $P$ be a polyomino without holes, having $n$ rows and $n$ columns, $n \geq 1$. Let $\mathcal{A}=\left(A_{1}, \ldots, A_{2(r+1)}\right)$ be the list of its vertices (i.e., corners of its boundary) ordered in a clockwise direction starting from the lowest leftmost vertex, with $A_{i}=\left(x_{i}, y_{i}\right)$. We say that $P$ is a permutomino if $\mathcal{P}_{1}=\left(A_{1}, A_{3}, \ldots, A_{2 r+1}\right)$ and $\mathcal{P}_{2}=\left(A_{2}, A_{4}, \ldots, A_{2 r+2}\right)$ represent two permutations of length $n$. Obviously, if $P$ is a permutomino, then $r=n$, see Fig. 4. As an immediate consequence we have that $m=n$, and so a permutomino has $n$ rows and $n$ columns ( $n$ will be called its size), and the number of its vertices is $2(n+1)$. The two sets $\mathcal{P}_{1}$ and $\mathcal{P}_{2}$ can be regarded as two permutation matrices of $[n+1]=\{1,2, \ldots, n+1\}$ having no common points; we indicate the permutations associated with them by $\pi_{1}$ and $\pi_{2}$, respectively (see Fig. 4). They are called the permutations associated with $P$.

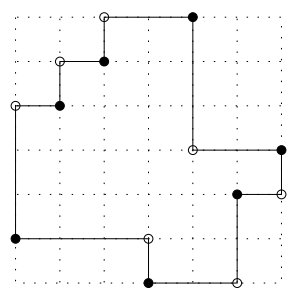

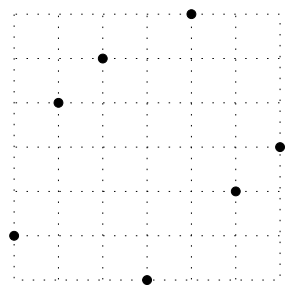

$\pi_{l}=(2,5,6,1,7,3,4)$

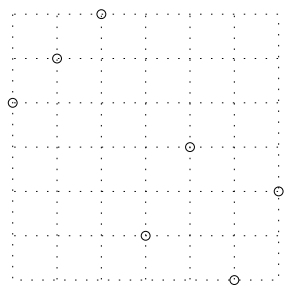

$\pi_{2}=(5,6,7,2,4,1,3)$

Fig. 4. A permutomino and the two associated permutations. The dotted black vertices represent the elements of $\mathcal{P}_{1}$, while the circled vertices the elements of $\mathcal{P}_{2}$. 
From the definition any permutomino $P$ of size $n$ has the property that, for each abscissa (ordinate) between 1 and $n$ there is exactly one vertical (horizontal) side in the boundary of $P$ with that coordinate. It is simple to observe that this property is also a sufficient condition for a polyomino to be a permutomino.

\section{Convex Permutominoes Tiling the Plane}

In this section we study convex permutominoes which are also pseudo-square polyominoes, that we call pseudo-square convex permutominoes (briefly, pscpermutominoes). They present interesting geometrical and combinatorial aspects.

First, we recall that we assume that the word $X$ encoding the boundary of a generic psc-permutomino has the form $N^{+}(E \vee N)^{*} N^{+}$. Moreover, we will work indifferently with the word or the path representation of $X$ and $Y$.

We say that a binary word $X$ is compatible with $Y$ if the word $X Y \bar{X} \bar{Y}$ represents the boundary of a psc-polyomino. So, for instance, we have that the word $X=N N E N N$ is compatible with $Y=E E N E E$ (see Fig. 5 (a)), with $Y^{\prime}=$ EESEE (see Fig. [5 (b)), and with $Y^{\prime \prime}=$ EESEESSEESSSEESSEESEE (see Fig. 5 (c)). A word $X$ of the form $N^{+}(E \vee N)^{*} N^{+}$is said to be exact if there is at least a word $Y$ which is compatible with $X$.

From the definition of permutomino and of pseudo-square, and from the convexity constraint, The following statement holds.

Proposition 2. For each word $X$ of the form $N^{+}(E \vee N)^{*} N^{+}$there exist at most two different words $Y$ and $Y^{\prime}$, having minimal length, compatible with $X$ such that:

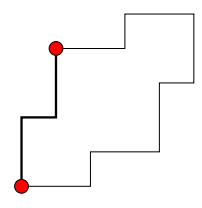

(a)

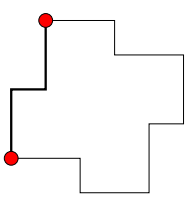

(b)

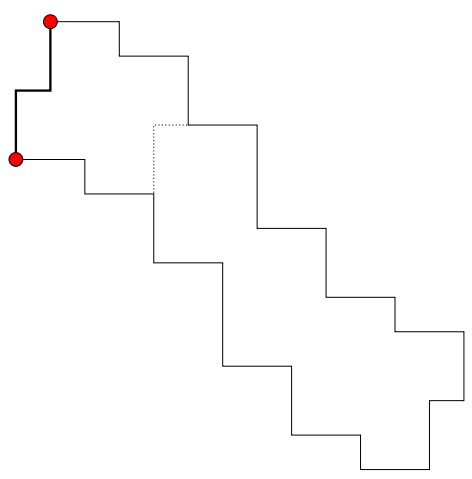

(c)

Fig. 5. Three $p s p$-permutominoes obtained from the same word $X=N N E N N$. The permutomino in $(a)$ has an upward growing direction, while those in $(b)$ and $(c)$ have a downward the growing direction. 
1. neither $Y$ nor $Y^{\prime}$ is a prefix of the other,

2. if there exists $Y^{\prime \prime}$ compatible with $X$, then either $Y$ or $Y^{\prime}$ is a prefix of $Y^{\prime \prime}$.

It is also clear that, in a pseudo-square convex permutomino, the word $X$ completely determines the related words $Y$ and $Y^{\prime}$. For instance referring to Figure 5 , the word $X=N N E N N$ is compatible with $Y=E E N E E$, and $Y^{\prime}=E E S E E$ while $Y^{\prime \prime}=Y^{\prime} S S E E S S S E E S S Y^{\prime}$.

The first classification of $p s c$-permutominoes is given by the concept of growing direction, which follows directly from Proposition 2 . We say that a pscpermutomino has upward growing direction if the word $Y$ has the form $E^{+}(N \vee$ $E)^{*} E^{+}$. Similarly, we say that it has downward growing direction if $Y$ has the form $E^{+}(S \vee E)^{*} E^{+}$(see Fig. [5). In the next sections we will study separately the psc-permutominoes having upward and downward growing direction. According to this, we say that $X$ and $Y$ are up-compatible (resp. down-compatible) with $X$ if $X Y \bar{X} \bar{Y}$ is a psc-permutomino having upward (resp. downward) growing direction.

\subsection{Up-Growing Direction: Pseudo-square Parallelogram Permutominoes}

The reader may have noticed that $p s c$-permutominoes having upward growing direction are precisely the pseudo-square parallelogram permutominoes (see Figure $5(\mathrm{a}))$. We observe that, starting from $X$, and given a positive integer $n$, there is a unique path $Y(n)$ of length $n$, and using steps $E$ and $N$, such that the object $X(n)$ obtained by concatenating $Y(n)$ at the beginning and at the end of $X$ has exactly one side for each abscissa and ordinate between 1 and $n$. Similarly, letting $n$ tend to infinity, we obtain a unique infinite path $Y_{\infty}$, and an object $X(\infty)$ having exactly one side for each abscissa and ordinate greater than 1 (see Fig. 6). In $X(n)$, the path $Y_{\infty}$ starting from the end (resp. beginning) of $X$ will be called the upper (resp. lower) path of $X(n)$. The following property is straightforward.

Proposition 3. Every word $Y$ which is up-compatible with $X$ is a prefix of $Y_{\infty}$.

In what follows, we prove that the word $Y_{\infty}$ is periodic, and we provide a simple algorithm to determine a prefix of length $n$ of $Y_{\infty}$ from $X$.

First, we start by recalling the definition of mex of positive (or empty) integer set $v$, noted $\operatorname{mex}(v)$ : it is the smallest positive integer of the complementary of the set $v$ in $\mathbb{N}$. For instance, if $v=\{0,1,4\}$ then the complement of $v$ is $\{2,3,5,6, \ldots\}$ thus $\operatorname{mex}(v)=2$. This definition appears in some works of A.S. Fraenkel and it is related to the Nim game, Sturmian and balanced sequences [4].

The basic idea for constructing the path $Y_{\infty}$ is to look separately at the horizontal and vertical steps in $X$.

The first step consists in building the vector $V$ whose $i$ th entry $V_{i}$ is given by the length of the $i$ th vertical segment in $X$, and the vector $H$, whose $i$ th entry is given by the length of the $i$ th horizontal segment in $Y$. So, for instance, if $X=N N E N N$ we have $V=(2,2)$ and $H=(1)$, see Fig. 6 (a). 


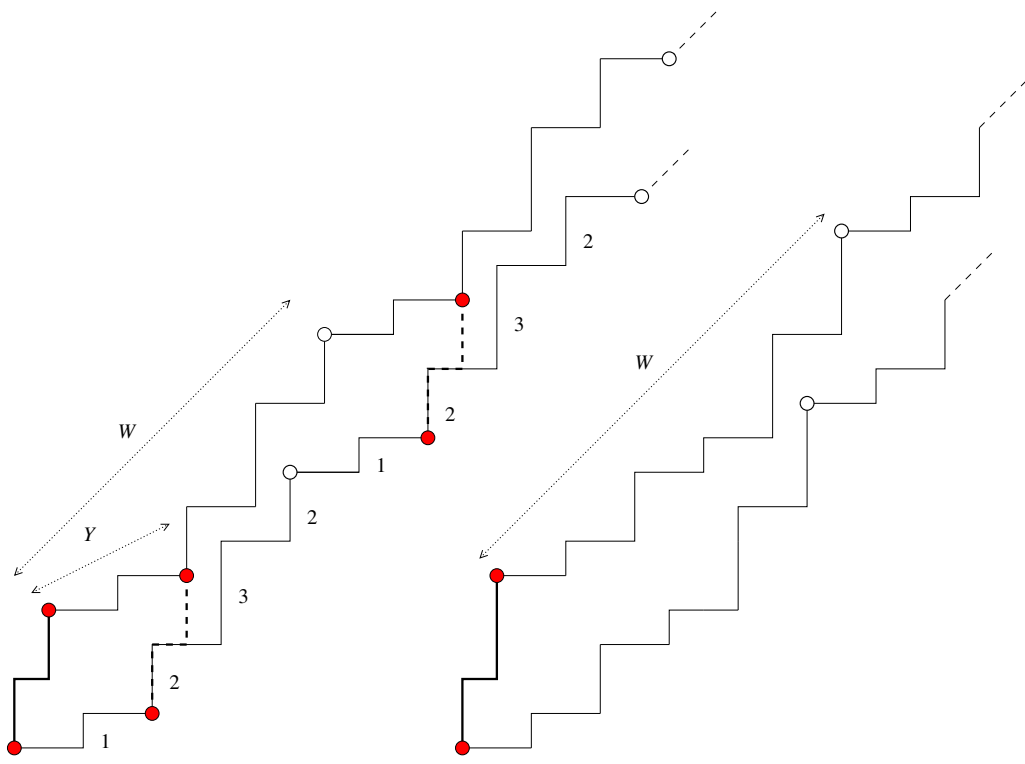

(a)

(b)

Fig. 6. The path $X(\infty)$ does not cross itself: $(a)$ we have no pseudo-square parallelogram permutomino; $(b)$ there is a word $Y$ of length less than the period $W$ of $Y_{\infty}$ such that $X$ is up-compatible with $Y$, and we have a pseudo-square parallelogram permutomino

Then we focus on the vertical steps growth, and for any given $i \geq 1$ we construct the integer set $v(i)$ by iteration, according to the following rule:

Base: $v(1)=\left\{0, V_{1}, V_{1}+V_{2}, V_{1}+V_{2}+V_{3}, \ldots\right\}$ : it is the set of the ordinates where the vertical segments of $X$ start or end; moreover, we set the variable $p(1)=0$.

Step: $v(i+1)=v(i) \cup \operatorname{mex}(v(i)) \cup \operatorname{mex}(v(i))-p(i)+\max (v(i))$, then we set $p(i+1)=\operatorname{mex}(v(i))$.

We also build the integer vector $V_{Y}$, whose $i$ th entry is given by the term $\operatorname{mex}(v(i-1))-p(i-1)$. Referring to our example, we have:

- $v(1)=\{0,2,4\}$, and $p(1)=0$

$-\operatorname{mex}(v(1))=1, \operatorname{mex}(v(1))-p(1)+\max (v(1))=5$, that is $v(2)=\{0,1,2,4,5\}$, and $V_{Y}=(1)$; now, $p(2)=\operatorname{mex}(v(1))=1$;

$-\operatorname{mex}(v(2))=3$ and $\operatorname{mex}(v(2))-p(2)+\max (v(2))=3-1+5=7$, then $v(3)=\{0,1,2,3,4,5,7\}$, and $V_{Y}=(1,2)$; now $p(3)=3$;

$-\operatorname{mex}(v(3))=6$ and $\operatorname{mex}(v(3))-p(3)+\max (v(3))=6-3+7=10$, that is $v(4)=\{0,1,2,3,4,5,6,7,10\}$, and $V_{Y}=(1,2,3)$; now, $p(4)=6$;

$-\operatorname{mex}(v(4))=8$ and $\operatorname{mex}(v(4))-p(4)+\max (v(4))=12$, that is $v(5)=$ $\{0,1,2,3,4,5,6,7,8,10,12\}$ and $V_{Y}=(1,2,3,2)$. 
We observe that the $i$-th element of $V_{Y}$ is just the length of the $i$ th vertical segment added in the construction of $Y_{\infty}$ (see Fig. 6 (a)). Now if we go on with the iteration, we observe that, for any $i>4, V_{Y}(i)=V_{Y}(j)$, where $j$ is simply the rest of the division of $i$ by 4 . This implies that $V_{Y}$ is periodic of period 4 . This means that the vertical segments in $Y_{\infty}$ are such that the length of the $i$-th vertical segment is equal to $V_{Y}(i \bmod 4)$.

Starting from $H$, and with an analogous algorithm, we can recursively build the set $h(i)$, and then the vector $H_{Y}$, whose $i$ th entry gives the length of the $i$ th horizontal segment in $Y_{\infty}$. Referring to our example, we have that $H_{Y}$ is periodic of length 2 , precisely $H_{Y}=(2)$. This means that all the horizontal steps in $Y_{\infty}$ have length 2 (see Fig. 6 (a)).

Using the previously defined tools, we are now ready to prove our main result.

Theorem 1. For any given $X=N^{+}(E \vee N)^{*} N^{+}$, there exists a positive integer $p$ dividing $4|X|_{N}|X|_{E}$ such that the infinite word $Y_{\infty}$ has period $p$.

Proof. To prove the periodicity of $Y_{\infty}$ it is sufficient to prove that the vectors $V_{Y}$ and $H_{Y}$ are periodic. First of all, let us make some remarks on the construction of $v(i)$ previously described. We recall that $p(i)=\operatorname{mex}(v(i-1))$ with the convention that $\operatorname{mex}(v(0))=0$. Thus there is an invariant in the construction: $\operatorname{mex}(v(i))-p(i)+\max (v(i))-\operatorname{mex}(v(i))=\max (v(i))-\operatorname{mex}(v(i-1))$. In fact, by construction we have $\max (v(i))=\operatorname{mex}(v(i-1))-p(i-1)+\max (v(i-1))$, then

$$
\begin{aligned}
\max (v(i))-\operatorname{mex}(v(i-1)) & =-p(i-1)+\max (v(i-1)) \\
& =\max (v(i-1))-\operatorname{mex}(v(i-2)) \\
& =\max (v(1))-\operatorname{mex}(v(0))=\max (v(1))
\end{aligned}
$$

In other words, at each step $i>0$ of the iteration we add to $v(i)$ two elements at distance $\max (v(1))$. In the path $Y_{\infty}$ that we are building, these two elements represent a vertical segment in the lower path, and a vertical segment in the upper path, respectively.

Now we would like to encode the set $v(i)$ by means of a vector $w(i)[0, \ldots$, $\max (v(i))]$ whose entries are the symbols $\{a, b, c, \cdot\}$, using the following:

i. for every element $j$ in the base set $v(1)$ we place the symbol $a$ in position $j$ of $w(1)$, and all other places are filled with $\cdot$,

ii. at the generic step $i+1$ of the iteration, the newly added element $\operatorname{mex}(v(i))$ is represented by placing $b$ in position $\operatorname{mex}(v(i))$ of $w(i+1)$, while the element $\operatorname{mex}(v(i))-p(i)+\max (v(i))$ is represented placing $c$ in position $\operatorname{mex}(v(i))-$ $p(i)+\max (v(i))$ of $w(i+1)$. We use the symbol $\cdot$ to say that a given position has not been filled yet by a $b$.

Thus, at each step we add two new symbols, $b$ and $c$, to the word $w(i-1)$, and they are always at distance $\max (v(1))$.

So referring to our example, $v(1)$ is represented by the word $w(1)=a \cdot a \cdot a$, then the set $v(2)=\{0,1,2,4,5\}$ is represented by $a b a \cdot a c$, since we add a 


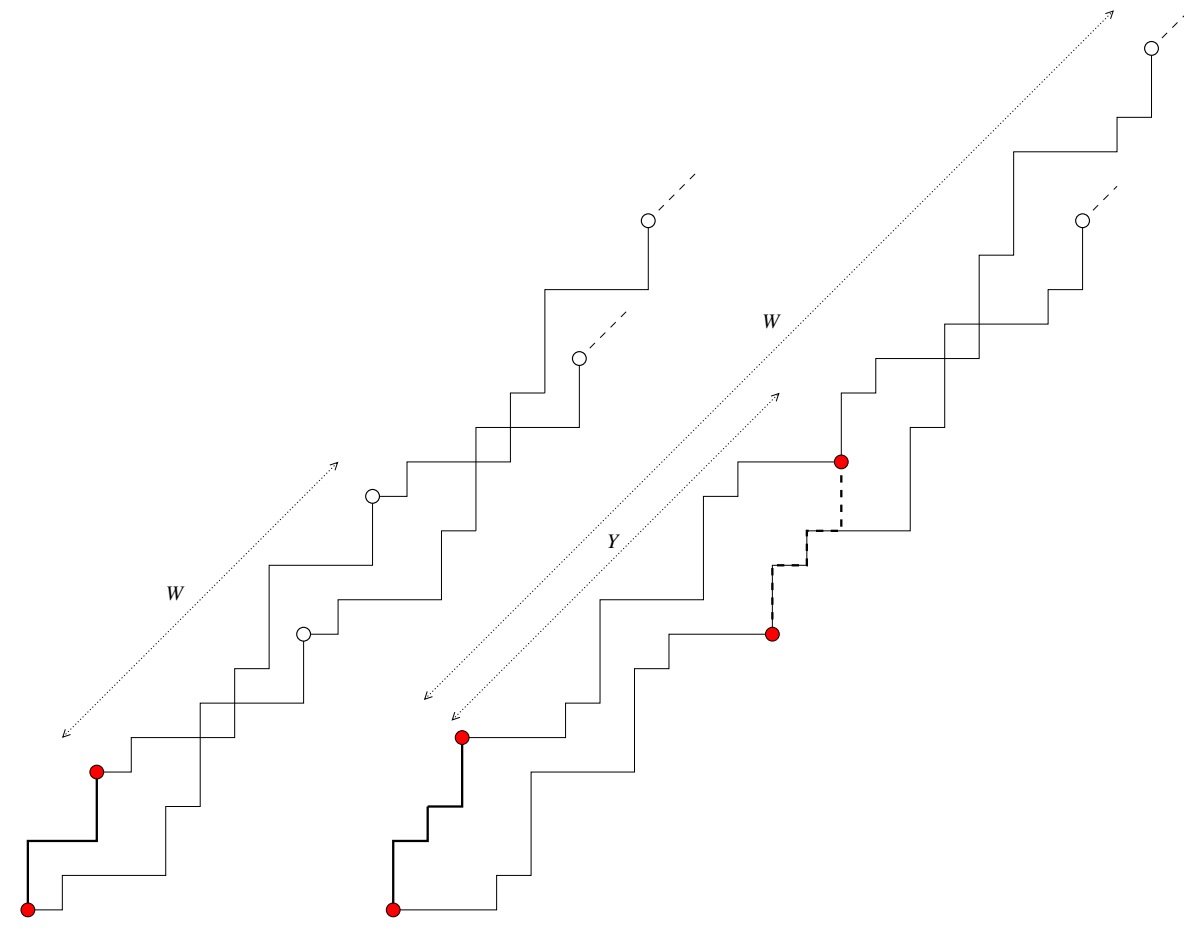

(a)

(b)

Fig. 7. The path $X(\infty)$ crosses itself: $(a)$ there is no pseudo-square parallelogram permutomino; (b) there is a word $Y$ of length less than the period $W$ of $Y_{\infty}$ such that $X$ is up-compatible with $Y$, and we have a pseudo-square parallelogram permutomino

$b$ in position 1 and a $c$ in position 5 , at distance $\max (v(1))=4$. Then the representation for $v(3)=\{0,1,2,3,4,5,7\}$ is $w(3)=a b a b a c \cdot c$. Successively we have $w(4)=a b a b a c b c \cdot c, w(5)=a b a b a c b c b \cdot c \cdot c$, and so on.

We can prove now that the element $2 \max (v(1))$ always represents a segment in the lower path and it is coded by $b$. Otherwise, by the invariant $2 \max (v(1))-$ $\max (v(1))=\max (v(1))$ should represent a vertical segment of the lower path, and this is impossible because $\max (v(1))$ is clearly coded by $a$.

Using the same argument: each $a$ of the word $w(1)$ in position $j>0$ is "translated" by $\max (v(1))$ to a $b$ in position $j+\max (v(1))$; each $b$ is position $j \geq 0$ of $w(i)$ is translated to a $c$ in position $j+\max (v(1))$, and similarly each $c$ is position $j \geq 0$ of $w(i)$ is translated to a $b$ in position $j+\max (v(1))$.

Thus, it's when the element in position $2 \max (v(1))$ is filled by a $b$ for the first time that we find the period. Indeed, let $i$ be the first index such that $w(i)$ has a $b$ in position $2 \max (v(1))$. Concerning the word $w(i)$ we have $a$ in position $0, a$ in position $\max (v(1))$ and $b$ in position $2 \max (v(1))$. For all other positions, $a$ in position $j$ implies $b$ in position $j+\max (v(1))$, and $c$ in position $j+2 \max (v(1))$. 


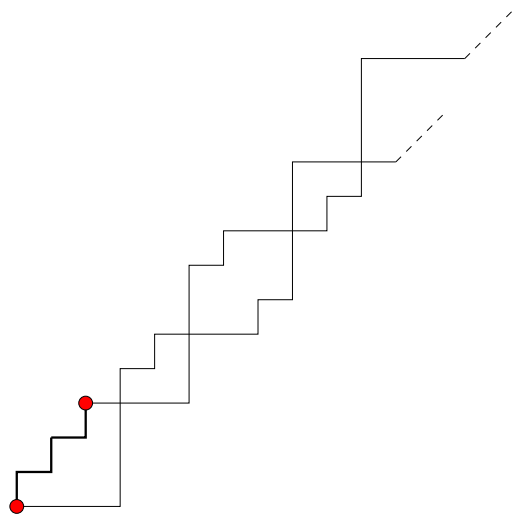

(a)

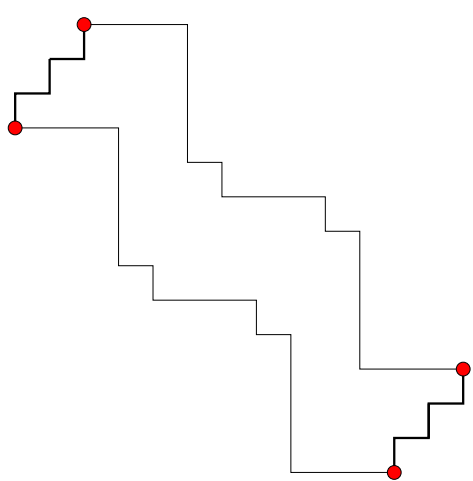

(b)

Fig. 8. (a) the path $Y_{\infty}$ : there is no word $Y$ which is up-compatible with $X ;(b)$ a prefix $Y^{\prime}$ of the path $Y_{\infty}^{\prime}$ which is down-compatible with $X$

By construction the $b$ in position $2 \max (v(1))$ is the last $b$ placed in $w(i)$, and all the positions on the right of it are either filled by a $c$ or by a dot. Moreover, all the other $c$ in position greater than $2 \max (v(1))$ have been translated from an $a$ in position $j$ by the translation $2 \max (v(1))$. Finally, there is a clear correspondence between the elements of $w(1)$ and those of $w(i)[2 \max (v(1)), \ldots, \max (v(i))]$, which leads to the periodicity. Referring to our example, we have $w(1)=a \cdot a \cdot a$, then $w(5)=a b a b a c b c \mid b \cdot c \cdot c$. In summary, we need $2|X|_{N}$ iterations to have periodicity on $V_{Y}$ f the vertical steps, while similarly, we need $2|X|_{E}$ iterations to have periodicity on $H_{Y}$. By mixing the two words, we have that $Y_{\infty}$ has pe$\operatorname{riod} 2|X|_{N} \cdot 2|X|_{E}=4|X|_{N}|X|_{E}$.

The proof of Theorem 1 gives us a simple recursive way to generate the infinite word $Y_{\infty}$ :

$-Y_{\infty}(0)=\emptyset$;

- $Y_{\infty}(i)=Y_{\infty}(i-1) E^{H_{Y}(i) \bmod \left|H_{Y}\right|} N^{V_{Y}(i) \bmod \left|V_{Y}\right|}$.

Then $Y_{\infty}$ is the limit of $Y_{\infty}(i)$ for $i \rightarrow \infty$.

From now on, let $X$ be given, let $p$ be the period of $Y_{\infty}$, and let $W=$ $Y_{\infty}[1, \ldots, p]$. We remark that the previously described algorithm gives us as a corollary a simple way to check if the boundary of $X(\infty)$ crosses itself or not. Since $Y_{\infty}$ is periodic, it is sufficient to check it for at most $p$ steps. Summarizing, concerning pseudo-square parallelogram permutominoes, we may have the following possibilities:

1. the boundary of $X(\infty)$ crosses itself. Then by Theorem 1 it crosses itself infinitely many times. In this case, we may have the two possibilities: $(a)$ we have no pseudo-square parallelogram permutomino (see Fig. 7 (a)); (b) there is a prefix $Y$ of $W$ which is up-compatible with $X$, and we have a pseudo-square parallelogram permutomino (see Fig. 7 (b)). 
2. the boundary of $X(\infty)$ does not cross itself; in this case, it may happen that there are no words $Y$ which are up-compatible with $X$ (see Fig. 6] (a)); otherwise, if there is at least a word $Y$ up-compatible with $X$, then there are infinitely many such words; assuming that $Y$ is the word having minimal length among these ones, then they have the form $W^{*} Y$ (see Fig. 6] (b), where the black points identify the paths $Y, W Y, W^{2} Y, \ldots$, and the white points identify the paths $\left.W, W^{2}, \ldots\right)$.

\section{Down-Growing Direction}

Most of the considerations made for the up-growing direction can be easily reformulated for the down-growing direction. Also in this case, for a given word $X$ of the form $N^{+}(E \vee N)^{*} N^{+}$, we can define an infinite word $Y_{\infty}^{\prime}$, and every word $Y^{\prime}$ which is down-compatible with $X$ is a prefix of $Y_{\infty}^{\prime}$. Moreover, we can write down an algorithm - completely analogous to that presented in the previous section - to recursively build the word $Y_{\infty}^{\prime}$. There is also a strict relation between the two words $Y_{\infty}$ and $Y_{\infty}^{\prime}$ which is exploited in the next statement.

Proposition 4. Let $X$ be a word of the form $N^{+}(E \vee N)^{*} N^{+}$. The following properties hold:

i) $Y_{\infty}^{\prime}$ is obtained from $Y_{\infty}$ by changing all $N$ steps with $S$ steps (see Fig. 8).

ii) The word $Y_{\infty}^{\prime}$ is periodic, and its period is a divisor of $4|X|_{N}|X|_{E}$.

iii) If $X$ is up-compatible with $Y$, then $X$ is down-compatible with the word $Y^{\prime}$, which is obtained from $Y$ by changing all $N$ steps with $S$ steps (see Fig. 5).

The converse of iii) however does not hold, for instance Fig. 5 shows that $X=$ $N E N E N$ is down-compatible with a word $Y^{\prime}$, while it is not up-compatible with the word $Y$ - obtained from $Y^{\prime}$ by changing all $S$ steps with $N$ steps - and there is no word $Y$ which is up-compatible with $X$.

\section{Further Work}

Using an exhaustive program written in SAGE we were led to formulate some conjectures, tested for $|X| \leq 13$. In a polyomino, the properties of being both a pseudo square and a permutomino determine strong symmetries of the word $X$, more precisely it seems that

Conjecture 1. If the word $X$ of the form $N^{+}(E \vee N)^{*} N^{+}$is exact, then it is a palindrome having odd length.

The reader can test this conjecture on all the examples presented throughout the paper. Assuming Conjecture 1, by symmetry, we have that each word $Y$ related to $X$ in the decomposition of $P$ as pseudo-square is a palindrome of odd length too. The converse of Conjecture 1 does not hold, as one can easily check with the word $X=N N N E N N N$. Our main goal would be to characterize the palindromic words which determine psc-permutominoes and then possibly 
enumerate these words according to the length. For instance, we have three exact words of length 5, NNENN (both in the up-growing and in the down-growing directions), $N E E E N$ and $N E N E N$ (only in the down-growing direction).

Another challenging and more general problem concerns the study of pseudohexagon convex permutominoes; in this case, as established in [1, many of the basic properties of pseudo-square convex polyominoes do not hold so we do not have unicity of the decomposition. Also the problem of studying non convex permutominoes which tile the plane by translation is completely open.

\section{References}

1. Brlek, S., Frosini, A., Rinaldi, S., Vuillon, L.: Tilings by translation: enumeration by a rational language approach. The Electronic Journal of Combinatorics 13(1) (2006)

2. Bernini, A., Disanto, F., Pinzani, R., Rinaldi, S.: Permutations defining convex permutominoes. Journal of Integer Sequences, J. Int. Seq. 10, Article 07.9.7 (2007)

3. Disanto, F., Frosini, A., Pinzani, R., Rinaldi, S.: A closed formula for the number of convex permutominoes. El. J. Combinatorics 14, \#R57 (2007)

4. Fraenkel, A.S.: The Rat game and the Mouse game, to appear in Games of No Chance 4

5. Girault-Beauquier, D., Nivat, M.: Tiling the plane with one tile. In: Proceedings of the Sixth Annual Symposium on Computational Geometry, Berkley, California, United States, June 7-9 (1990)

6. Golomb, S.W.: Polyominoes: Puzzles, Patterns, Problems, and Packings. Princeton Academic Press, London (1996)

7. Kassel, C., Lascoux, A., Reutenauer, C.: The singular locus of a Schubert variety. J. Algebra 269, 74-108 (2003)

8. Wijshoff, H.A.G., Van Leeuven, J.: Arbitrary versus periodic storage schemes and tesselations of the plane using one type of polyomino. Inform. Control 62, 1-25 (1991) 